\title{
Kinetics of walnut shells through Pyrolysis Assessed as an alternative Bio-fuel
}

\author{
Mudasir Akbar Shah, N. S.Khana, Vimal Kumar
}

\begin{abstract}
Biomass residues have potential to be used as an alternative solid fuel for various processes, also will be responsible for the optimization of the pyrolysis reaction processes, giving insight into the pyrolysis mechanisms and aiding in engineering efforts. There are different methods for production of bio-fuel from biomass residues; however before the production of bio-fuels from biomass it is important to understand their thermal degradation and kinetics. In the present work the Kinetics studies of four different walnut shells, namely, paper (PSW), thin (TSW), medium (MSW) and hard (HSW) shelled walnuts have been studied. Thermal degradation behaviour of walnut shells is investigated using thermogravimetric analysis (TGA) under three different heating rates $\left(30,60\right.$ and $\left.100^{\circ} \mathrm{Cmin}^{-1}\right)$ in an oxidizing atmosphere to understand the kinetic behavior. Kissinger-Akahira-Sunose (KAS) and Ozawa-Flynn-Wall (OFW) methods were used to estimate the kinetic parameters such as Pre-exponential factor $(R)$ and average activation energy $\left(E_{a}\right)$ using TGA data of active zone. The average activation energy of biomass obtained from PSW, TSW, MSW and HSW using KAS method were 198.25, 184.21, 180.93, and $152.79 \mathrm{~kJ} \mathrm{~mol}^{-1}$, respectively. Whereas by $O F W$ method the values were found to be $204.10,186.28,188.39$ and $153.78 \mathrm{~kJ} \mathrm{~mol}^{-1}$ respectively. Thus the present investigation may be useful for its use as a renewable energy source and potential candidate for fuel production by thermo-chemical processes.
\end{abstract}

Keywords: Different types of walnut shells; Kinetic parameters; Thermo gravimetric analysis (TGA).

\section{INTRODUCTION}

Biomass is considered to be the most abundant renewable alternate energy source evenly distributed in the world. It is an attractive option for power generation. The use of fossil fuels in power plants can be substituted by biomass without any major transitional economic adjustment. Continuous depletion of fossil fuels, rising prices of petroleum, increasing demand for energy, and environmental problems are the critical reasons due to which there is much emphasis on alternative renewable energy sources. Sustainability, biodegradability and environmental friendliness are the valuable features which have made the biomass as dominant

Revised Manuscript Received on June 10, 2020.

* Correspondence Author

Mudasir Akbar Shah*, Department of Chemical Engineering, National Institute of Technology Srinagar, J \& Kr-190006, India E-mail: shahmudasir22@gmail.com

N. S. Khana, Department of Chemical Engineering, National Institute of Technology Srinagar, J \&Kr-190006, India.

Vimal Kumar, Department of Chemical Engineering, Indian Institute of Technology Roorkee, Uttarakhand-247667, India.

(C) The Authors. Published by Blue Eyes Intelligence Engineering and Sciences Publication (BEIESP). This is an open access article under the CC BY-NC-ND license (http://creativecommons.org/licenses/by-nc-nd/4.0/) source for production of bio-energy. Another important feature of biomass is that it has very low amount of ash, nitrogen and sulfur contents which make the biomass more attractive from the stand point of environment [1]. Walnut shell is one of the agricultural biomass waste materials obtained from the dry fruit, walnut and belongs to the group of nutshells. The main producer of walnut is mainly in Europe, North America and Asia can be used to produce chemicals such as activated carbons, lignin and carbon molecular sieve $[2,3]$ It consists of $60 \%$ kernel (the oily material) and $40 \%$ shell (the hard covering of the walnut $[\underline{4}, \underline{5}, \underline{6}]$. The kernel, which comes out when cracked, is used for various purposes, whereas the shell remains mostly unused. The shell is marginally utilized for heating purpose by directly combusting into furnaces. However, it can be utilized for the production of bio-fuels or high value added chemicals for suitable processes. India is the seventh walnut producing country in the world, where Jammu and Kashmir contributes about $98 \%$ of the country's total walnut output. In the year 2016-2017, about 266,280 Mts was produced in Jammu and Kashmir [7]. As reported by Global Agricultural Information Network, the walnut production in India is expected to increase by three percent to reach 34,000 MT in 2017-18 [8] Walnut production in India is primarily confined to the hilly states of Jammu and Kashmir, Uttarakhand and Himachal Pradesh [8]. The area, production and productivity of walnut in India from 20010-11 to 2016-17 have been shown in the Table I. According to one report, $38 \%$ of the world's production of walnut shell is from United States, from which 99\% is produced in California [9]. With the expansion of walnut, the amount of walnut wastes-walnut shells has increased markedly.

Table-I: All Indian Area, Production and Productivity of walnut

\begin{tabular}{|l|l|l|l|}
\hline Year & $\begin{array}{l}\text { Area } \\
\text { in '000 HA) }\end{array}$ & $\begin{array}{l}\text { Production } \\
\text { (in '000MT) }\end{array}$ & $\begin{array}{l}\text { Productivity } \\
\text { (in MT/HA) }\end{array}$ \\
\hline $2010-11$ & 137 & 201 & 1.5 \\
\hline $2011-12$ & 172 & 288 & 1.7 \\
\hline $2012-13$ & 144 & 242 & 1.7 \\
\hline $2014-15$ & 115 & 238 & 2.1 \\
\hline $2015-16$ & 92 & 229 & 2.5 \\
\hline $2016-17$ & 122 & 228 & 2.4 \\
\hline
\end{tabular}

Extensive studies are available in the literature on the thermo-gravimetric analysis and kinetic parameter estimation during thermal degradation of biomass residues $[\underline{10}, \underline{11}, \underline{12}$, $13,14,15,16]$. Braz and Crankovic studied the kinetics of six different biomass residues like tucumã seed, coffee husk, peanut shell, sugar cane bagasse,

Blue Eyes Intelligence Engineering 


\section{Kinetics of walnut shells through Pyrolysis Assessed as an alternative Bio-fuel}

pine sawdust and rice husk [17]. It was reported that, at the initial stage of the thermal degradation tucuma seed have lower activation energy and it increases monotonously with the reaction, which is an indication of the easy ignition of the fuel. However, for other biomass samples the TGA profile showed stable behaviour.

Also, different biomass samples showed the presence of oxygen, which indicated the ignition processes even in the absence of air. The kinetic parameter estimation studies are broadly classified to model fitting and model free or also referred as "iso-conversional" methods. In case of model fitting methods different models are fitted with experimental data to get the best statistical fit model to estimate the kinetic parameters. The model fitting methods produce highly unreliable kinetic parameter values when applied to non-isothermal data. These problems can be addressed by using iso-conversional methods, where kinetic curves are plotted at different heating rates to estimate the kinetic parameters irrespective of reaction function and order. Iso-conversional methods have been used both for single as well as multiple step processes to describe the kinetics of thermal degradation processes $[\underline{10}, \underline{11}, \underline{12}]$. From these studies it was concluded that the iso-conversional methods, such as Flynn-Wall-Ozawa (OFW), Kissinger-Akahira-Sunose (KAS) and Coats-Redfern, are more reliable methods to estimate kinetic parameters of a biomass: activation energy $\left(E_{a}\right)$ and pre-exponential factor (A). In iso-conversional methods the basic assumptions are (i) for a constant conversion the reaction rate depends on the temperature only, and (ii) the reaction mechanisms are independent of the heating rate. Therefore, model free methods are easy in application and predict more reliable values of kinetic parameters for different biomass samples during thermal decomposition. From latest trend, large investigation are going on for efficient and economical method of biomass conversion technologies and its utilization were reported in literatures like Combustion, pyrolysis, liquefaction and gasification. Pyrolysis is the most attractive process for converting biomass into energy [1, 19, 20], however, due to highly complex mechanism that is influenced by many factors, like properties of raw materials and the operating conditions [21, 22]. By using TG repeatable data collection has the advantage of pyrolysis rate, which makes a deep investigation of the kinetic parameters [23, 24]. Different researchers have used these methods to calculate the kinetic parameters from TGA data of pyrolysis of various biomass and waste materials such as karanj fruit hulls, hazelnut husk, and apple pomace [25, 26, 27]. Walnut shell may be utilized in many areas. Presently, its primary application is the production of activated carbon and as a pyrolysis feedstock. It is reported that walnut shell filters are usually installed for removing solid matter and oils produced from water bodies, refineries and from other industries. The basic properties which make pulverized walnut shell preferable for filtration is its adsorbent properties. A study conducted to estimate the efficiency of walnut shell as adsorbent reported that up to $96 \%$ oil removal may be achieved [28]. Investigations have shown that the activated carbon produced from walnut shell was found economically feasible and environmentally friendly [29]. It may also use for varieties of industrial applications. These include cleaning and de flashing, as fillers in manufacturing, for blasting polishing etc [30]. Now day's research scientists are more interested in finding out good feedstock for pyrolysis or for other conversion technologies. Walnut shell may be a potential feed stock for producing chemical or fuels.

In the present work the kinetic parameter estimation during thermal decomposition of different types of walnut shells have been carried out. The thermal decomposition processes were evaluated for different walnut shell samples available in India: paper (PSW), thin (TSW), medium (MSW) and hard (HSW) shelled walnuts, using oxidizing (air) atmosphere. The kinetics parameters were determined by using Kissinger-Akahira-Sunose (KAS) and Flynn-Wall-Ozawa (OFW) models. The optimal kinetics mechanism was determined by the maximum correlation coefficients, activation energies and frequency factors calculated by using iso-conversional methods by using TGA data in active zone. The study results clearly shows biomass, can be used as alternative solid fuel for various processes, also will be responsible for the optimization of the pyrolysis reaction processes, giving insight into the pyrolysis mechanisms and aiding in engineering efforts.

\section{MATERIALS AND METHODS}

\subsection{Material}

Four types of walnut shells with particle size $0.1 \mathrm{~mm}$ : paper (PSW), thin (TSW), medium (MSW) and hard (HSW) shelled walnuts were collected from the local walnut industry Srinagar Kashmir, India. Fine powdered material was packed in air tight PVC containers and stored in desiccators for further use in the present research.

\subsection{TG Analysis}

Thermo-gravimetric analysis (TGA) was carried out by the thermogravimetric analyser (SII TG/DTA 6300 instrument). The tests were conducted non-isothermally over a wide range of temperature i.e., 28 to $1000{ }^{0} \mathrm{C}$, at three different heating rates $\left(30,60\right.$ and $100{ }^{\circ} \mathrm{Cmin}^{-1)}$ in oxidizing atmosphere at a flow rate of $200 \mathrm{mLmin}^{-1}$. Biomasses were placed on open platinum sample pans during the TG analysis. For each experiment a sample mass of $10 \pm 0.26 \mathrm{~g}$ were taken. The instrument was used to measure and record the changes in mass of the samples with temperature. Small sample size favours good temperature uniformity during determination.

\subsection{Models Developed for Kinetic Analysis of different types of Biomass}

Kinetic study is very essential for understanding the nature of thermal decomposition of biomass and getting the best kinetics model for kinetic parameters estimation. The kinetic model considered in the present work is described below.

In order to calculate the kinetic parameters by using TGA data, there are three iso- conversional models suchas: (i). Coats-Redfern methods ii). Kissinger-Akahira-Sunose method (KAS) and (iii). Ozawa-Flynn-Wall method (OFW) these models are most reliable and widely accepted used to calculate the kinetic parameters like activation energy $\left(E_{\mathrm{a}}\right)$, the reaction model $f(\alpha)$ and correlation factor (R). KAS and OFW models were used by different researchers like [31, 32, 33]. Whereas Coats-Redfern method was used to investigate the reaction mechanism likes diffusion, contacting chemical reaction and geometry [25].

Published By:

Blue Eyes Intelligence Engineering

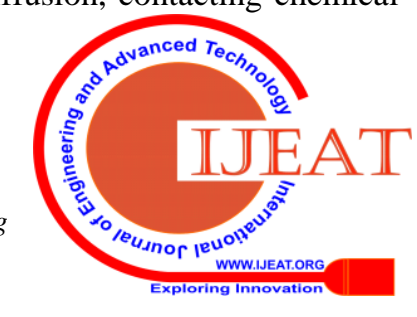


In thermogravimetric analysis, Biomass decomposition process can be generally expressed as:

Biomass (solid) $\longrightarrow$ Bio-yields (bio-char/oil and Gases) Solid mass decomposition commonly described as:

$$
\frac{\mathrm{d} \alpha}{\mathrm{d} t}=k \mathrm{f}(\alpha)
$$

Where $\alpha, t$ and $k$ refer to fractional change in conversion, rate constant and $f(\alpha)$ refers to reaction model, respectively. Equation (1.1) signifies the rate of conversion as a function of temperature and conversion. The fractional change was calculated using equation (1.2), as follows:

$$
\alpha=\frac{m_{\mathrm{i}}-m_{\mathrm{t}}}{m_{\mathrm{i}}-m_{\mathrm{f}}}
$$

Where, $m_{\mathrm{i}}, m_{t}$ and $m_{\mathrm{f}}$ are the initial mass, mass at a time $t$, and final mass of the biomass residues, respectively. The rate constant $k$ is generally given by Arrhenius eq.

$$
k=\mathrm{Ae}^{\left(-E_{\mathrm{a}} / R T\right)}
$$

Where $A, E_{\mathrm{a}}, T$ and $R$ are pre-exponential factor, activation energy, absolute temperature and universal gas constant (8.314 J/K.mol), respectively. On combining equations 1.1 and 1.3 we have

$$
\frac{\mathrm{d} \alpha}{\mathrm{d} t}=\mathrm{A} \mathrm{e}^{\left(-E_{\mathrm{a}} / R T\right)} \mathrm{f}(\alpha)
$$

For dynamic case temperature is dependent both on time and heating rates and described as

Follows, Where $\beta=d T / d t$, Introducing the heating rate into equation. 1.4, we obtain,

$$
\frac{\mathrm{d} \alpha}{\mathrm{d} T}=\frac{\mathrm{A}}{\beta} \mathrm{e}^{\left(-E_{\mathrm{a}} / R T\right)} f(\alpha)
$$

In order to calculate kinetic parameters by using analytical methods on the given TGA data, rearrange the equation 1.5

$$
\frac{\mathrm{d} \alpha}{f(\alpha)}=\frac{\mathrm{A}}{\beta} \mathrm{e}^{\left(-E_{\mathrm{a}} / R T\right)} \mathrm{d} T
$$

Now using initial conditions of $\alpha=0$ and $\mathrm{T}=\mathrm{T}_{0}$ After integration and rearranging equation (1.6), Kissinger formula was developed and expressed as follows:

$$
\ln \left(\frac{\beta}{T^{2}}\right)=\log \left[\frac{A E_{\mathrm{a}}}{R g(\alpha)}\right]-\frac{E_{\mathrm{a}}}{R T}
$$

Using equation (1.7) the activation energy can be evaluated from the plot of $\ln \left(\beta / T^{2}\right)$ versus $1 / T$ with slope $-\frac{E_{\mathrm{a}}}{R}$ gives a straight line.

The activation energy was also estimated by Flynn- WallOzawa (OFW) model as:

$\ln \beta=\ln \left[\frac{A E_{\mathrm{a}}}{R g(\alpha)}\right]-2.315-0.457 \frac{E_{\mathrm{a}}}{R T}$

\section{WhPERE}

$$
\mathrm{g}(\alpha)=\ln (1-\alpha) / T^{2}
$$

for $n=1$

$$
=\left(1-(1-\alpha)^{1-n} /(1-n) T^{2}\right.
$$

Plot, $\ln (\alpha)$ versus $1 / \mathrm{T}$ for three heating rates (30, 60, 100 ${ }^{0} \mathrm{C} / \mathrm{min}$ ) and for conversion (0.1 to 0.8 ) values corresponding to activation energy should be parallel line with slope of $-0.457 E_{a} / R$. The Coats-Fern method is derived from Arrhenius equation and is used for estimating the reaction mechanism at particular reaction order (n) in addition to activation energy and pre-exponential factor of the sample. We get

$\ln g(\alpha)=\ln \frac{A R}{\beta E_{\mathrm{a}}}-\frac{E}{R T}$

\section{RESULTS AND DISCUSSION}

\subsection{Thermo-gravimetric analysis}

Thermal behaviour was studied and the TG curves for four different walnut shells are shown in Figure.1 (i-iv). From Fig.1 (i-iv) it can be seen that the thermal decomposition of four different samples can be divided in to three stages due to variable slope curves. In the first stage, i.e. up to $120-190{ }^{\circ} \mathrm{C}$, the mass loss was due to the free and sorbet water vaporization. This zone constitutes an approximately a mass loss of $7-12 \%$. The present finding is in agreement with the work of Aciklin and $\operatorname{Kar}[\underline{34}, \underline{35}]$. In the TG curve the main devolatilization step begin at about $\approx 200{ }^{\circ} \mathrm{C}$ and complete at about $\approx 375{ }^{\circ} \mathrm{C}$ in terms of heating rates with an average mass loss of $55 \%$. This step indicates the decomposition of hemicelluloses and cellulose, which are the major components in biomass. The mass loss is high in this stage and therefore is referred as active pyrolysis zone. After $420{ }^{0} \mathrm{C}$ passive pyrolysis zone started and went on up to $800{ }^{\circ} \mathrm{C}$ with a mass loss about $16 \%$ for all walnut shell samples.

The thermal decomposition behaviours can be explained by different components present in walnut shell, which is mainly comprised of lignocelluloses materials. Thermo-gravimetric behaviour of hemicelluloses, cellulose and lignin components has been studied by [36], and their decomposition was reported within the temperature range of 210-325, 310-400 and $160-900{ }^{\circ} \mathrm{C}$, respectively. In active pyrolysis zone the different reactions observed may be due to the decomposition of cellulose and hemicellulose. The nature of thermal decomposition for the four walnut shell samples were analysed at 30, 60 and $100{ }^{0} \mathrm{C} / \mathrm{min}$ (Figure 1 Thermal decomposition profiles of different walnut shell were similar, which is due to the similar composition of all walnut samples considered. However, the HSW requires a little higher temperature to vaporize the physically absorbed water than other samples (TSW, MSW and PSW) due to its hard structure.

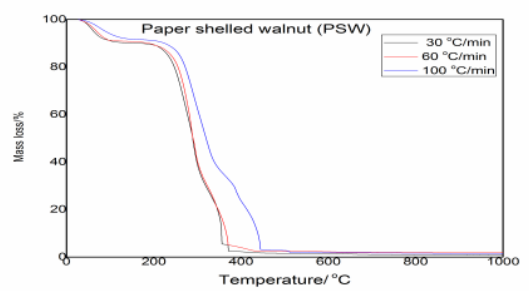

(i)

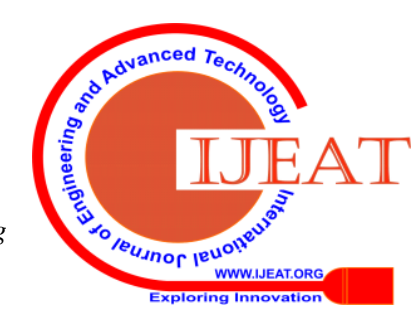




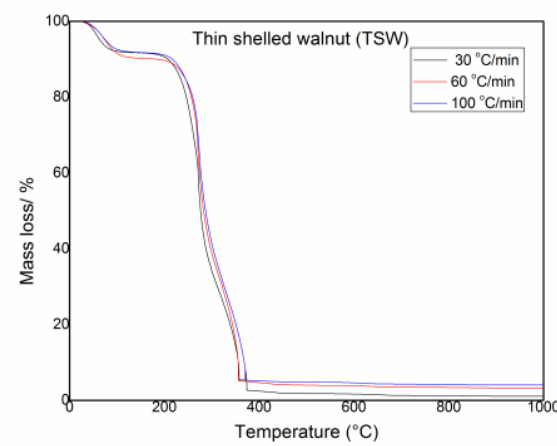

(ii)

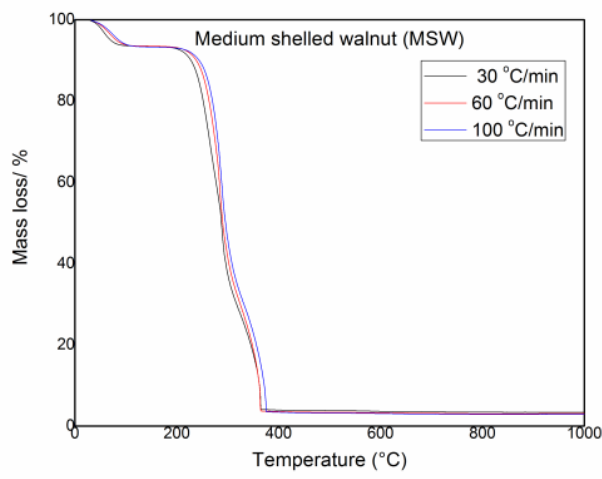

(iii)

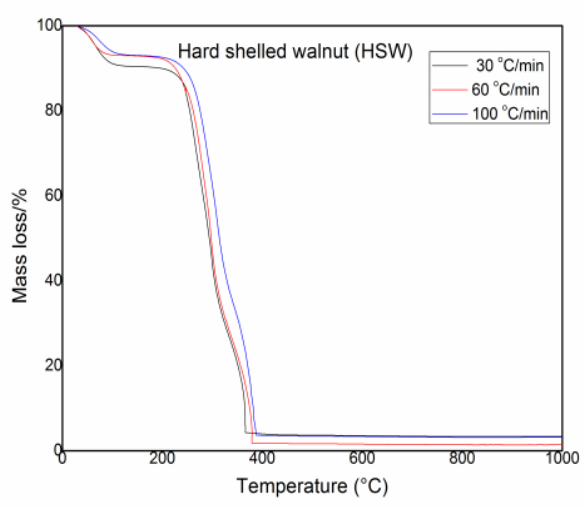

(iv)

Fig. 1.TGA profile of (i) PSW (ii) TSW (iii) MSW and (iv) $\mathrm{HSW}$ in air atmosphere at $\left(30,60\right.$ and $\left.100{ }^{\circ} \mathrm{C} / \mathrm{min}\right)$ heating rates.

\subsection{Kinetic behaviour of Walnut shells}

The kinetic parameters (activation energy and correlation factor) obtained were estimated by KAS and OFW and Coats-Redfern methods. The active pyrolysis zone of the biomass residues ranging from $\left(190-375{ }^{\circ} \mathrm{C}\right)$ was considered for the analysis. Curves derived heating rates (30, 60 and 100 ${ }^{0} \mathrm{C} / \mathrm{min}$ ) with degree of conversion $(\alpha)$ ranging from 0.1 to 0.8 in an oxidizing atmosphere was taken for the kinetic study of different samples. Figure 2 and Figure 3 show the kinetic plots of PSW, TSW, MSW and HSW with KAS and OFW models, respectively. It can be seen that the fitted lines at different conversions for all types of walnut shells were nearly parallel with each other, which indicated that there is a possibility of single reaction mechanism.

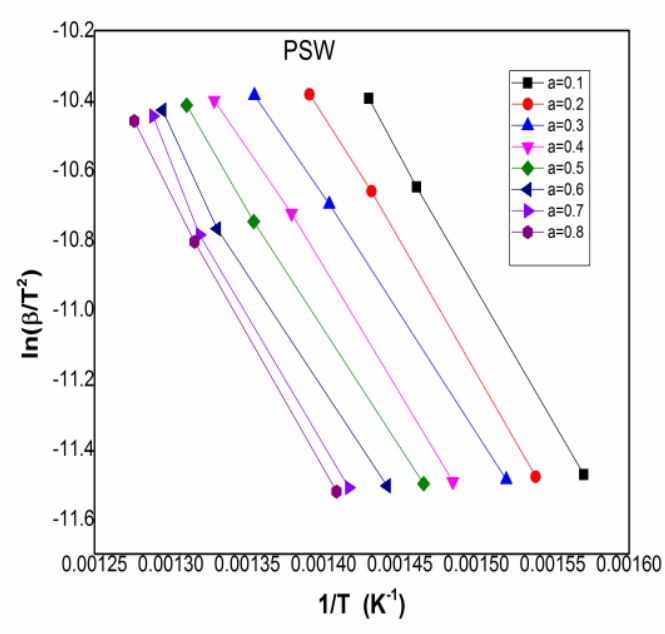

(i)

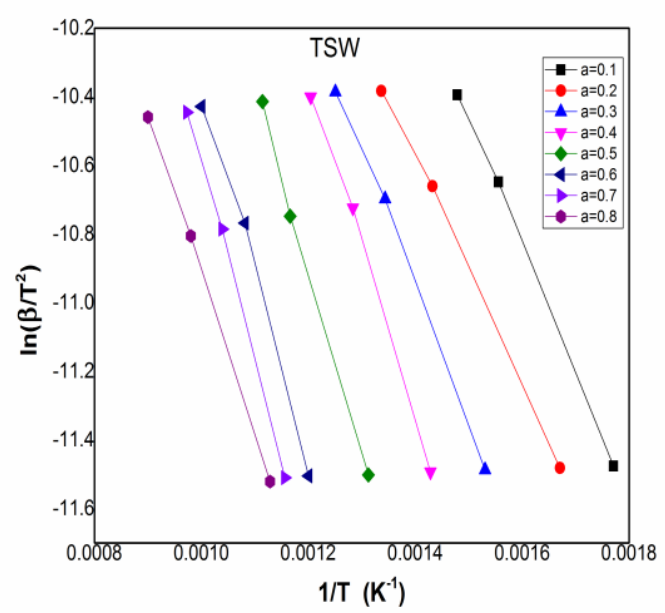

(ii)

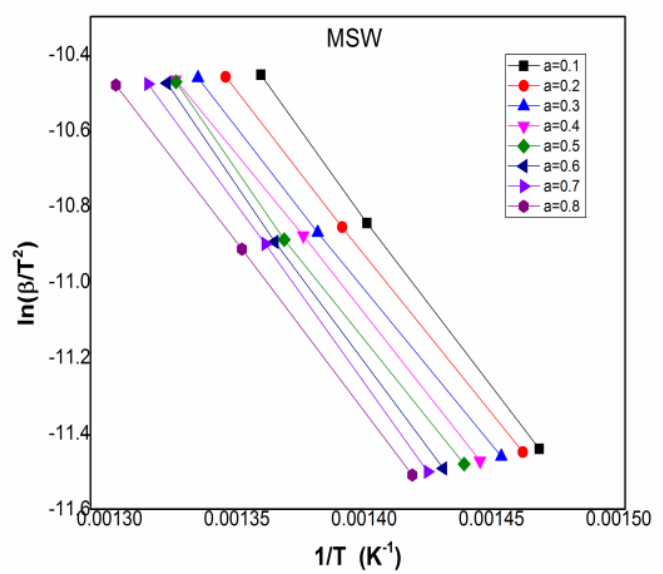

(iii)

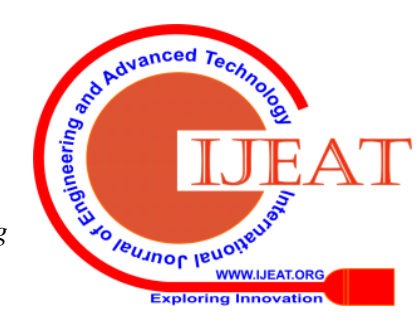




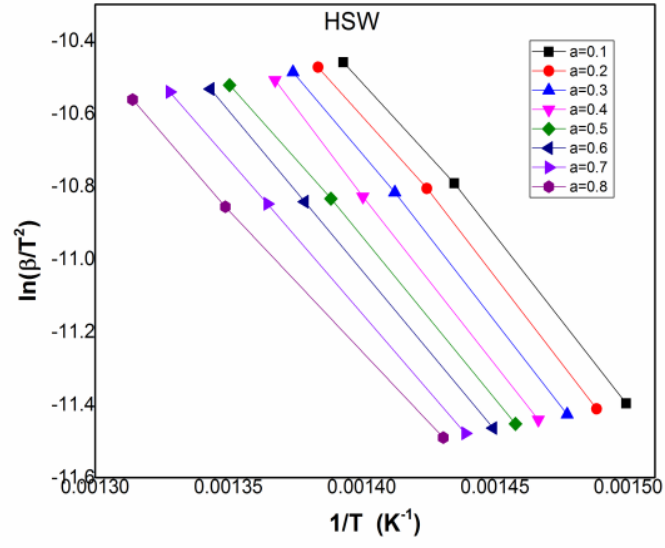

(iv)

Fig. 2. Kinetic plots of (i) PSW (ii) TSW (iii) MSW and (iv) HSWobtained by the KAS method

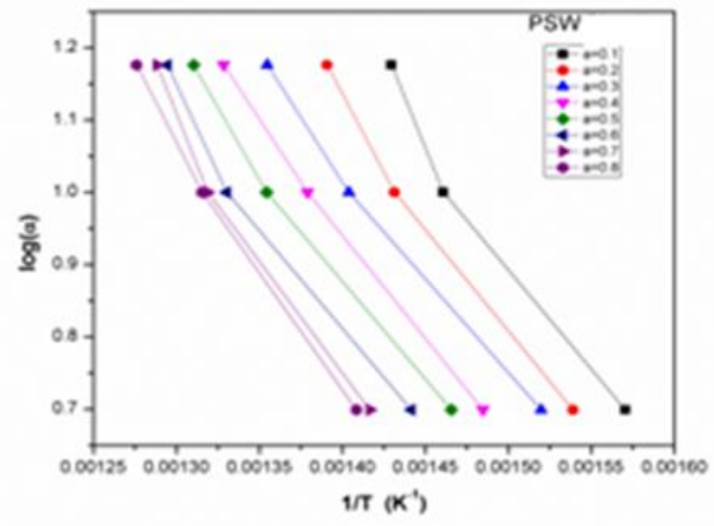

(i)

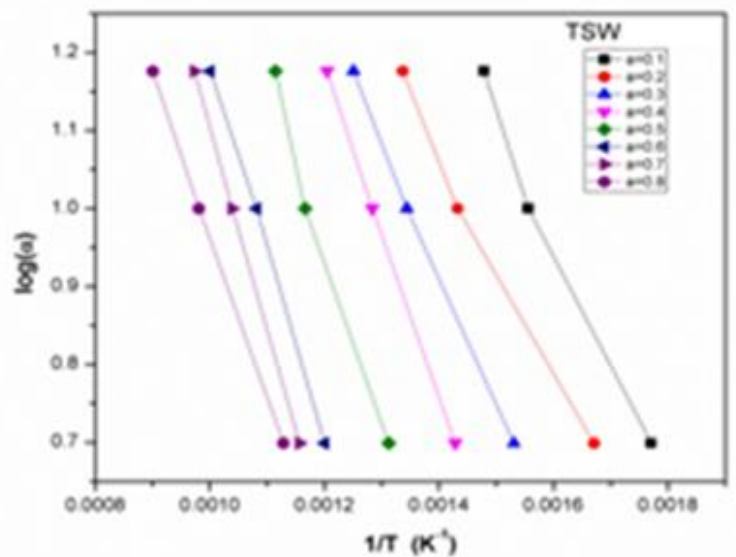

(ii)

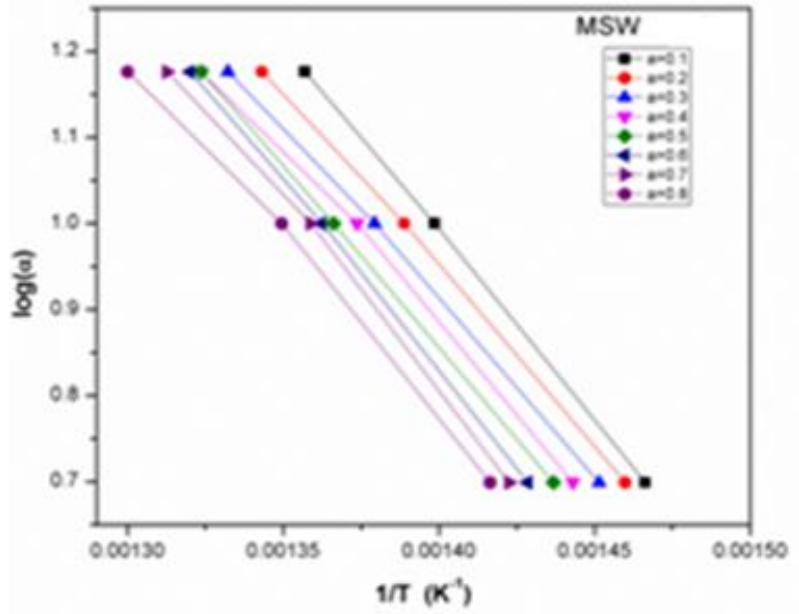

(iii)

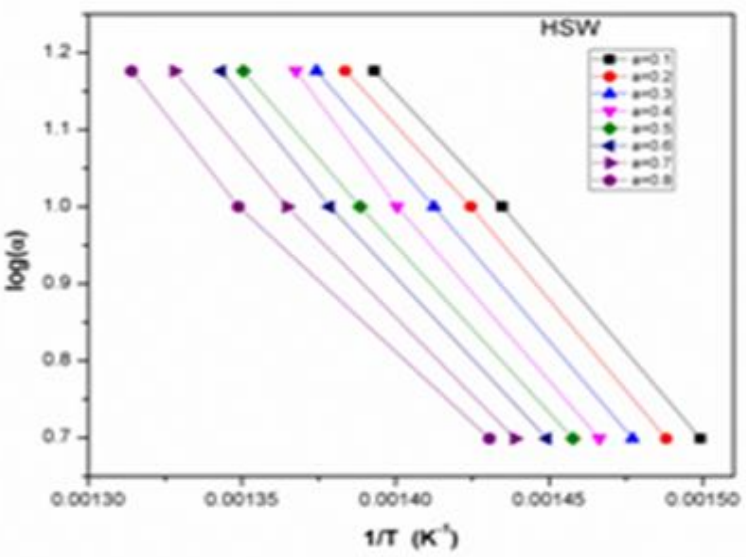

(iv)

Fig. 3. Kinetic plots of (i) PSW (ii) TSW (iii) MSW and (iv) HSW obtained by the OFW method.

The average activation energy value from KAS method for different walnut shell were 198.25, 184.21, 180.93, and $152.79 \mathrm{~kJ} / \mathrm{mol}$ and for OFW 204.78, 186.28, 188.39 and $153.78 \mathrm{~kJ} \mathrm{~mol}^{-1}$ for (PWS), (TWS), (MWS) and (HWS) respectively, are listed in Table II and Table III. It was observed that the activation energy first increased with an increase in conversion (0.1-0.2), then decreased (0.2-0.3) and finally increased from 0.3 conversions onwards. It may be due to the removal of moisture and light volatile matters in the initial stage, the activation energy is low which then increased due to the conversion of complex molecules such as hemicellulose, cellulose and lignin. High values of activation energy indicated that the sample was thermally more stable since higher energy is needed during thermal-degradation process. The comparison of the activation energy estimated in the present work for different walnut shells have been carried out with the value of activation energies reported in the literature for different walnut shells. It can be seen from Table IV that there is wide variation in the values of activation energy reported in the literature, which may be due to the different thermal decomposition stages considered during different studies.

Blue Eyes Intelligence Engineering

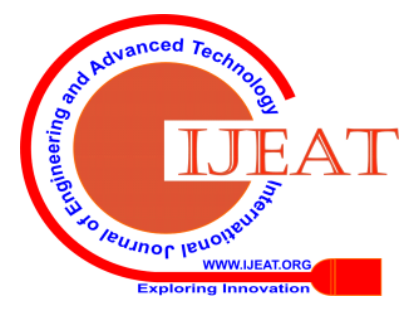


Kinetics of walnut shells through Pyrolysis Assessed as an alternative Bio-fuel

However, present predictions were in good agreement with walnut shells. the work of Yuan and Liu [30] for thermal-decomposition of

Table-II: Activation energy and correlation factor with different conversion by KAS methods.

\begin{tabular}{|c|c|c|c|c|c|c|c|}
\hline$\alpha$ & Equation & $R^{2}$ & $\begin{array}{c}E_{a} \\
\left(\mathrm{~kJ} \mathrm{mo}^{-1}\right)\end{array}$ & $\alpha$ & Equation & $R^{2}$ & $\begin{array}{c}E_{a} \\
\left(\mathrm{~kJ} \mathrm{mo}^{-1}\right)\end{array}$ \\
\hline & \multicolumn{3}{|c|}{ Paper shelled walnut(PSW) } & & \multicolumn{3}{|c|}{ Thin shelled walnut(TSW) } \\
\hline 0.1 & $y=-20301 x+29.65$ & 0.996 & 160.45 & 0.1 & $y=-20910 x+30.16$ & 0.999 & 168.30 \\
\hline 0.2 & $y=-22440 x+31.94$ & 0.999 & 184.10 & 0.2 & $y=-21063 x+28.40$ & 0.997 & 170.32 \\
\hline 0.3 & $y=-21188 x+27.82$ & 0.999 & 170.46 & 0.3 & $y=-19944 x+25.34$ & 0.999 & 165.81 \\
\hline 0.4 & $y=-22200 x+29.06$ & 0.999 & 182.08 & 0.4 & $y=-20611 x+25.67$ & 0.994 & 166.47 \\
\hline 0.5 & $y=-23188 x+29.25$ & 0.999 & 188.15 & 0.5 & $y=-20523 x+24.79$ & 0.992 & 167.43 \\
\hline 0.5 & $y=-25100 x+31.83$ & 1.0 & 204.10 & 0.6 & $y=-22100 x+26.81$ & 0.994 & 174.37 \\
\hline 0.7 & $y=-27200 x+34.44$ & 0.998 & 220.30 & 0.7 & $y=-23160 x+29.23$ & 0.998 & 195.95 \\
\hline \multirow[t]{3}{*}{0.8} & $y=-35200 x+45.96$ & 0.993 & 276.38 & 0.8 & $y=-29236 x+40.96$ & 0.993 & 265.0 \\
\hline & \multicolumn{2}{|c|}{ Average activation energy $\left(E_{a}\right)$} & \multicolumn{2}{|c|}{198.25} & \multicolumn{2}{|c|}{ Average activation energy $E_{a}$ ) } & 184.21 \\
\hline & \multicolumn{2}{|c|}{ Average correlation factor $\left(R^{2}\right)$} & \multicolumn{2}{|c|}{0.998} & \multicolumn{2}{|c|}{ Average correlation factor $R^{2}$ ) } & 0.996 \\
\hline$\alpha$ & \multicolumn{3}{|c|}{ Medium shelled walnut(MSW) } & $\alpha$ & \multicolumn{3}{|c|}{ Hard shelled walnut(HSW) } \\
\hline 0.1 & $\mathrm{Y}=-20580 \mathrm{X}+30.94$ & 0.998 & 173.42 & 0.1 & $y=-17769 x+23.16$ & 0.995 & 147.73 \\
\hline 0.2 & $Y=-20115 X+27.08$ & 0.999 & 160.82 & 0.2 & $y=-16643 x+19.87$ & 0.997 & 138.37 \\
\hline 0.3 & $\mathrm{Y}=-18023 \mathrm{X}+23.66$ & 0.998 & 151.16 & 0.3 & $y=-16805 x+19.43$ & 0.995 & 139.72 \\
\hline 0.4 & $\mathrm{Y}=-17810 \mathrm{X}+24.33$ & 0.998 & 160.95 & 0.4 & $y=-17357 x+19.72$ & 0.988 & 144.31 \\
\hline 0.5 & $Y=-20121 X+24.63$ & 0.992 & 159.8 & 0.5 & $y=-18370 x+20.80$ & 0.999 & 152.73 \\
\hline 0.6 & $\mathrm{Y}=-21912 \mathrm{X}+28.04$ & 0.99 & 189.60 & 0.6 & $=-18993 x+21.17$ & 0.995 & 157.91 \\
\hline 0.7 & $\mathrm{Y}=-23811 \mathrm{X}+32.01$ & 0.988 & 211.97 & 0.7 & $y=-20483 x+22.93$ & 0.997 & 170.30 \\
\hline \multirow[t]{3}{*}{0.8} & $\mathrm{Y}=-29045 \mathrm{X}+37.61$ & 0.995 & 239.79 & 0.8 & $y=-20596 x+22.51$ & 0.999 & 171.24 \\
\hline & \multicolumn{2}{|c|}{ Average activation energy $\left(E_{a}\right)$} & 180.93 & & \multicolumn{2}{|c|}{ Average activation energy $\left(E_{a}\right)$} & 152.79 \\
\hline & \multicolumn{2}{|c|}{ Average correlation factor $\left(R^{2}\right)$} & 0.995 & & \multicolumn{2}{|c|}{ Average correlation factor $\left(R^{2}\right)$} & 0.996 \\
\hline
\end{tabular}

Published By:

Blue Eyes Intelligence Engineering

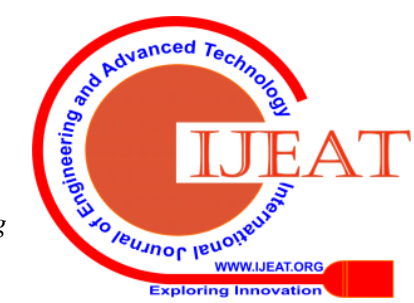


Table-III: Activation energy and correlation factor with different conversion by OFW methods.

\begin{tabular}{|c|c|c|c|c|c|c|c|}
\hline$\alpha$ & Equation & $\boldsymbol{R}^{2}$ & $\begin{array}{c}E_{a} \\
\left(\mathrm{kJJ} \mathrm{mo} 0^{-1}\right)\end{array}$ & $\alpha$ & Equation & $R^{2}$ & $\begin{array}{c}E_{a} \\
\left(\mathrm{~kJ} \mathrm{mo}^{-1}\right)\end{array}$ \\
\hline & \multicolumn{3}{|c|}{ Paper shelled walnut(PSW) } & & \multicolumn{3}{|c|}{ Thin shelled walnut(TSW) } \\
\hline 0.1 & $y=-20619 x+27.74$ & 0.974 & 169.01 & 0.1 & $y=-21582 x+30.492$ & 0.986 & 178.98 \\
\hline 0.2 & $y=-21995 x+30.51$ & 0.989 & 188.23 & 0.2 & $y=-20495 x+28.025$ & 0.991 & 168.29 \\
\hline 0.3 & $y=-22136 x+25.517$ & 0.989 & 178.00 & 0.3 & $y=-19203 x+30.283$ & 0.998 & 154.16 \\
\hline 0.4 & $y=-22308 x+29.094$ & 0.99 & 185.46 & 0.4 & $y=-20235 x+26.730$ & 0.999 & 166.67 \\
\hline 0.5 & $y=-23004 x+30.094$ & 0.99 & 195.67 & 0.5 & $y=-21326 x+27.752$ & 0.986 & 172.56 \\
\hline 0.5 & $y=-23004 x+28.094$ & 0.99 & 205.65 & 0.6 & $y=-23016 x+29.580$ & 0.998 & 182.88 \\
\hline 0.7 & $y=-27539 x+35.678$ & 0.993 & 226.98 & 0.7 & $y=-25963 x+31.696$ & 0.999 & 217.67 \\
\hline \multirow[t]{3}{*}{0.8} & $y=-35239 x+43.678$ & 0.993 & 289.23 & 0.8 & $y=-30181 x+34.046$ & 0.999 & 249.32 \\
\hline & \multicolumn{2}{|c|}{ Average activation energy $\left(E_{a}\right)$} & \multicolumn{2}{|c|}{204.78} & \multicolumn{2}{|c|}{ Average activation energy $\left(E_{a}\right)$} & 186.28 \\
\hline & \multicolumn{2}{|c|}{ Average correlation factor $\left(R^{2}\right)$} & \multicolumn{2}{|c|}{0.996} & \multicolumn{2}{|c|}{ Average correlation factor $\left(R^{2}\right)$} & 0.994 \\
\hline$\alpha$ & \multicolumn{3}{|c|}{ Medium shelled walnut(MSW) } & $\alpha$ & \multicolumn{3}{|c|}{ Hard shelledwalnut(HSW) } \\
\hline 0.1 & $y=-20437 x+29.112$ & 0.999 & 173.25 & 0.1 & $Y=-17988 \mathrm{X}+24.464$ & 0.999 & 151.24 \\
\hline 0.2 & $y=-21710 x+28.688$ & 0.997 & 176.32 & 0.2 & $Y=-17745 \mathrm{X}+20.519$ & 0.999 & 142.76 \\
\hline 0.3 & $y=-19588 x+25.534$ & 0.999 & 164.91 & 0.3 & $\mathrm{Y}=-16840 \mathrm{X}+18.554$ & 0.995 & 136.78 \\
\hline 0.4 & $y=-20401 x+26.499$ & 0.996 & 172.92 & 0.4 & $\mathrm{Y}=-17394 \mathrm{X}+20.725$ & 0.998 & 147.45 \\
\hline 0.5 & $y=-20422 x+26.764$ & 0.999 & 169.34 & 0.5 & $\mathrm{Y}=-19443 \mathrm{X}+19.164$ & 0.999 & 148.48 \\
\hline 0.6 & $y=-22441 x+27.002$ & 0.999 & 184.23 & 0.6 & $\mathrm{Y}=-19468 \mathrm{X}+22.554$ & 0.998 & 161.91 \\
\hline 0.7 & $y=-24366 x+29.917$ & 0.996 & 201.88 & 0.7 & $\mathrm{Y}=-20269 \mathrm{X}+23.838$ & 0.988 & 167.30 \\
\hline \multirow[t]{3}{*}{0.8} & $y=-33236 x+38.543$ & 0.995 & 266.23 & 0.8 & $Y=-20708 \mathrm{X}+23.444$ & 0.993 & 174.34 \\
\hline & \multicolumn{2}{|c|}{ Average activation energy $\left(E_{a}\right)$} & \multicolumn{2}{|c|}{188.39} & \multicolumn{2}{|c|}{ Average activation energy $\left(E_{a}\right)$} & 153.78 \\
\hline & \multicolumn{2}{|c|}{ Average correlation factor $\left(\mathrm{R}^{2}\right)$} & \multicolumn{2}{|c|}{.997} & \multicolumn{2}{|c|}{ Average correlation factor $\mathrm{K}^{2}$ ) } & .996 \\
\hline
\end{tabular}

Table-IV: Comparison of activation energy of various biomasses reported in the literature with the present study

\begin{tabular}{|l|l|l|}
\hline Biomass & $\begin{array}{l}\mathbf{E}_{\mathbf{a}}(\mathbf{k J} / \mathbf{m o l}) \\
\text { KAS method }\end{array}$ & \multirow{2}{*}{ References } \\
\cline { 1 - 2 } Paper shelled walnut (PSW) & 198.25 & Present \\
Thin shelled walnut (TSW) & 184.21 & \\
\cline { 1 - 2 } Medium shelled walnut (MSW) & 180.93 & \\
\cline { 1 - 2 } Hard shelled walnut(HSW) & 152.79 & {$[30]$} \\
\hline Walnut shell & $120-155$ & {$[34]$} \\
\hline Walnut shell & $45.6-78.4$ & {$[11]$} \\
\hline Wheat straw & $130-175$ & {$[16]$} \\
\hline Sugarcane bagasse & $126.62-148.80$ & {$[13]$} \\
\hline Cardoon steams & 224.51 & {$[17]$} \\
\hline Rice husk & 206 & \\
\hline
\end{tabular}

Figure 4 and Figure 5 show that the activation energy increases with an increase in conversion, which may be due to the change in reaction mechanism with an increase in temperature. The higher activation energy values were obtained in the pyrolysis zone. Also, the variation in conversion for different walnut shells was predicted and averaged at different heating rates of 30,60 and $100{ }^{\circ} \mathrm{C} / \mathrm{min}$, as shown in Figure 4 and Figure 5, It can be seen that for all samples the conversion (average) begins at $\approx 240{ }^{0} \mathrm{C}$ temperature, attain maximum at a temperature of $\approx 375{ }^{0} \mathrm{C}$ for heating rate of $30{ }^{0} \mathrm{C} / \mathrm{min}$. It was also observed that in the initial stage of thermo-chemical reaction, at a temperature of $200{ }^{0} \mathrm{C}$, there was $10 \%$ conversion at a heating rate of 30 ${ }^{0} \mathrm{C} / \mathrm{min}$. Further, in the active pyrolysis zone, i.e. temperature ranging from 190 to $375{ }^{0} \mathrm{C}$, the conversion was $80 \%$. Similarly, for a heating rate of $100{ }^{\circ} \mathrm{C} / \mathrm{min}$, the conversion initiates at $280{ }^{\circ} \mathrm{C}$ and attain maximum at $420{ }^{\circ} \mathrm{C}$. It indicates that the increase in heating rate during pyrolysis results in the higher temperature requirements for biomass decomposition. The difference of activation energy of various types of samples are due to number of factors, such as properties of biomass, pyrolysis conditions, experimental operating conditions, quantity and ash composition.

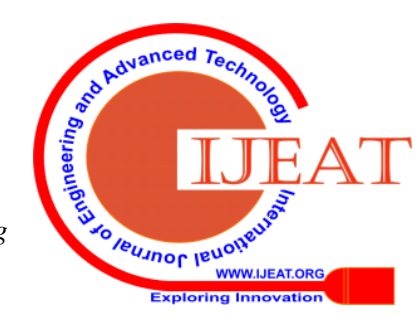




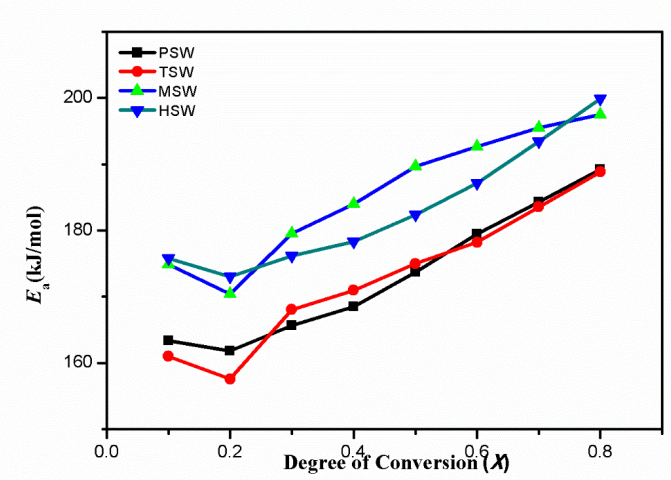

Fig. 4. Variations in activation energy with conversion of (i) PSW (ii) TSW (iii) MSW and (iv) HSW for KAS method.

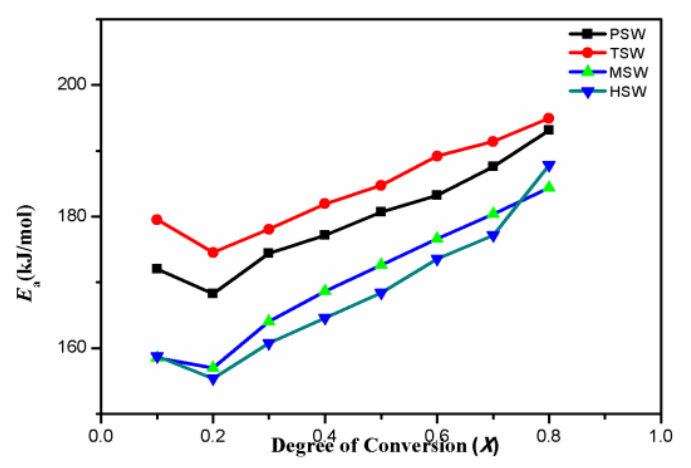

Fig. 5. Variations in activation energy with conversion of (i) PSW (ii) TSW (iii) MSW and (iv) HSWfor OFW method.

The pre-exponential factors, as a function of conversion, were estimated using Coats-Redfern method (equation 1.8). The activation energy values obtained from KAS and OFW methods were used in equation (1.8) for predicting pre-exponential factors, since both methods predicts reliable values of activation energy. The plot between $\ln \left[-\ln (1-\alpha) / T^{2}\right]$ and $1 / T$ at $\mathrm{n}=1$ resulted into a slopes $\left(-E_{\alpha} / R\right)$ and intercept $\left(\ln \left[A R / \beta E_{a}\right]\right)$. The values for pre-exponential factors (A) were obtained by inserting the values of heating rates $(\alpha)$, universal gas constant $(R)$ and activation energy $\left(E_{a}\right)$ in the intercept. Table 5 shows the values of pre-exponential factors obtained during thermo-chemical decomposition of biomass at different heating rates. It was observed that the pre-exponential factor values increased with an increase in heating rate. All the walnut shell samples showed similar values, with minor difference, of pre-exponential factor.

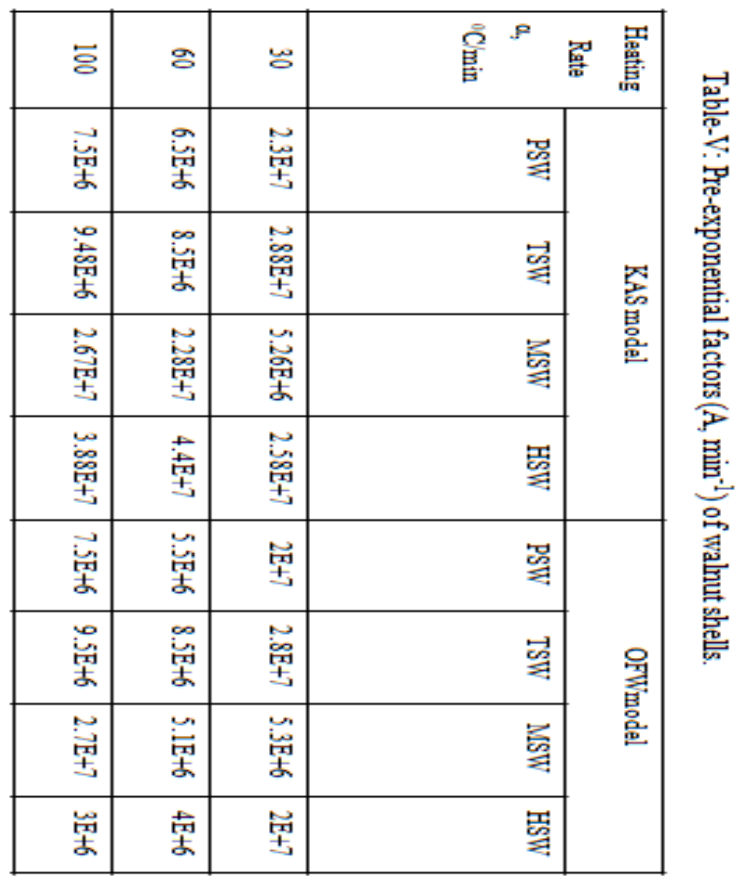

\section{CONCLUSIONS}

In this study, the thermal decomposition behavior of four different walnut shell samples was studied using KAS and OFW models. The average activation energy has been resulted between $179-204 \mathrm{~kJ} / \mathrm{mol}$ for all different samples. Thermogravimetric curves showed three distinct zones that can be associated to removal of water, decomposition of cellulose and hemicellulose and decomposition of lignin, respectively. In general, kinetics studies of biomass samples are a deep and vast concept, which require careful attention in fact needs a well speculated assumption. In the present work the model free methods was taken as a best alternative to study the walnut shell thermal decomposition. The kinetic parameters obtained by these two models were found in good agreement with values reported in the literature. The pre-exponential factor was estimated by Coats-Fern methods using activation energy value obtained with KAS and OFW models. The kinetic parameters obtained by these two models were found in good agreement with values reported in the literature. It was also observed that the values of activation energy for walnut shells were comparable with other biomass residues. Therefore, it seems that the Indian walnut shells have a promising potential for bio-fuel production through thermochemical conversion.

\section{REFERENCES}

1. A. Demirbas, "Combustion characteristics of different biomass fuels,". vol.30, Progress in Energy and Combustion Science, 2004, pp. 219-230.

2. E.V. Mathias, H.P. Halkar,"Separation and characterization of lignin compounds from the walnut (Juglans regia) shell oil using preparative TLC, GC-MS and1H NMR.,vol 71, Journal of Analytical and Applied Pyrolysis,2004, pp. 515-524.

3. E.David, A.Talaie, Stanciu,(2004) "Synthesis of carbon molecular sieves by benzene pyrolysis over microporous carbon materials", Journal of Materials Processing Technology,2004, pp. 157-158.

4. Global agricultural Information network (GIAN). Report number IN5116; 2016

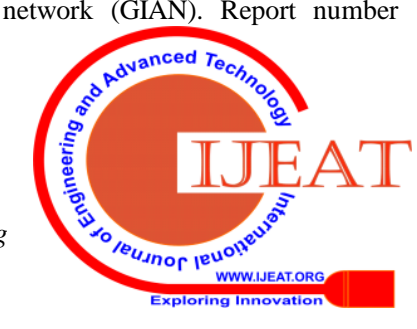


5. S.P.Malhotra, World edible nuts economy. ISBN, 2008, 13-978-81-8069-561-2.

6. M.A. Shah, M.N.S. Khan, V. Kumar,"Biomass residue characterization for their potential application as biofuels", vol, 134 Journal of Thermal Analysis and Calorimetry,pp.2137-2145.

7. M.H.Crops ( 2016-17) District-Wise / Kind -wise estimated Area under Major Horticulture Crops J \& K 2016-17 ( Area in Ha ) District-Wise / Kind -wise Production under major Horticulture Crops in J \& K State for the Year ) ( Production in Mts ), 17.

8. Global agricultural Information network (GIAN) Report Number.9-202017;

IN7113. https://gain.fas.usda.gov/Recent\%20GAIN\%20Publications/Tree\%20 Nuts\%20Annual_New\%20Delhi_India_9-20-2017.pdf http://nhb.gov.in/statistics/Publication/Horticulture\%20At\%20a\%20 Glance\%202017\%20for\%20net\%20uplod\%20(2).pdf

9. Walnut Production Manual University of California (1998) Division of Agriculture and Natural Resources. 3373.

10. A.K. Varma, P.Mondal, "Physicochemical characterization and kinetic study of pine needle for pyrolysis process", vol, 124 Journal of Thermal Analysis and Calorimetry,2016,pp.487-497.

11. J.M.Cai, L.S.Bi, "Kinetic analysis of wheat straw pyrolysis using isoconversional methods",vol,98, Journal of Thermal Analysis and Calorimetry,2009, pp.325-330.

12. K. AçIkalIn, "Pyrolytic characteristics and kinetics of pistachio shell by thermogravimetric analysis", vol,109, Journal of Thermal Analysis and Calorimetry,2012,pp.227-235.

13. T.Damartzis, D.Vamvuka, S. fakiotakis, "Thermal degradation studies and kinetic modeling of cardoon (Cynara cardunculus) pyrolysis using thermogravimetric analysis (TGA)", Bioresource Technology,2011, pp. 6230-6238.

14. R. Findorák, M. Fröhlichová, Legemza, "'Thermal degradation and kinetic study of sawdusts and walnut shells via thermal analysis",vol 125, Journal of Thermal Analysis and Calorimetry, 2016, pp.689-694.

15. F. Bai, Y. Sun, Y. Liu, (2016) "Thermogravimetric Analysis of Huadian Oil Shale Combustion at Different Oxygen Concentrations", vol, 30, Energy and Fuel,2016, pp.4450-4456.

16. E.R.Zanatta, T.O. Reinehr, Awadallak, "Kinetic studies of thermal decomposition of sugarcane bagasse and cassava bagasse", vol,125 Journal of Thermal Analysis and Calorimetry,2016, pp.437-445.

17. C.E.M. Braz, P.M.Crnkovic,"Physical - Chemical Characterization of Biomass Samples for Application in Pyrolysis Process ", vol,37, Chemical Engineering TransactionS,2014, pp.523-528.

18. M.Stenseng, A.Jensen, K.Dam-johansen, "Investigation of biomass pyrolysis by thermogravimetric analysis and differential scanning calorimetry", 2011, pp,765-780.

19. L.T.Vlaev, I.G. Markovska, L.A. Lyubchev, "Non-isothermal kinetics of pyrolysis of rice husk",vol, 406, Thermochimica Acta, 2003,pp.1-7.

20. Y.H.Lai, M.X.Lu, Y.C.Ma, "Research on pyrolysis characteristics of agricultural residues under liner heating temperature",vol,7 Journal of Combustion Science and Technology, 2001,pp.247-248

21. B.V.Babu, A.S.Chaurasia, "Pyrolysis of biomass: Improved models for simultaneous kinetics and transport of heat, mass and momentum",vol, 45, Energy Conversion and Management,2004, pp.1297-1327.

22. B.V.Babu, A.S.Chaurasia, "Modeling simulation and estimation of optimum parameters in pyrolysis of biomass",vol,44, Energy Conversion and Management,2003,pp. 2135-2158.

23. J.A.Caballero, J.A.Conesa, Font, "Pyrolysis kinetics of almond shells and olive stones considering their organic fractions",vol,42, Journal of Analytical and Applied Pyrolysis, 1997, pp.159-175.

24. P. Stolarek, S. Ledakowicz, "Pyrolysis kinetics of chitin by non-isothermal thermogravimetry",vol,433, Thermochimica Acta,2005, pp. 200-208.

25. J.E.White, W.J.Catallo, B.L. Legendre, "Biomass pyrolysis kinetics: A comparative critical review with relevant agricultural residue case studies", Journal of Analytical and Applied Pyrolysis, 2011, pp. 91:1-33.

26. M.A. Islam, M. Asif, B.H.Hameed, "Pyrolysis kinetics of raw and hydrothermally carbonized Karanj (Pongamia pinnata) fruit hulls via thermogravimetric analysis",vol,179, Bioresource Technology,2015,pp.227-233.

27. M.R.B.Guerrero, D.S.P.Marques, M. Zaragoza,"Thermogravimetric study on the pyrolysis kinetics of apple pomace as waste biomass", vol, 39, International Journal of Hydrogen Energy,2014, pp.16619-16627.

28. Z.Z. Ismail, "Removal of Oil From Wastewater Using Walnut-Shell", Al-Khwarizmi Engineering Journal, 2005,pp. 117-124.

29. B.Wookeun, K.Jongho, C. Jinwook, "Production of granular activated carbon from food processing wastes( walnut shells and jujube seeds) and its Adsorptive properties", vol,64, Journal of the air and waste management association,2014, pp.879-886

30. Yuan and Liu,"Study on pyrolysis Kinetics of walnut shell", vol, 89, Journal of Thermal Analysis and Calorimetry,2004,pp.983-986.

31. S.A.El-Sayed, M.E.Mostafa, " Kinetic Parameters Determination of Biomass Pyrolysis Fuels Using TGA and DTA Techniques",vol,6, Waste and Biomass Valorization,2015,pp. 401-415.

32. S.Ceylan, Y.Topçu, "Pyrolysis kinetics of hazelnut husk using thermogravimetric analysis", vol,156, Bioresource Technology,2015, pp.182-188.

33. A.V.Bridgwater, "Review of fast pyrolysis of biomass and product upgrading", VOL,38, Biomass and Bioenerg,2012, pp 68-94.

34. K. Açıalkn, "Thermogravimetric analysis of walnut shell as pyrolysis feedstock",vol,105, Journal of Thermal Analysis and Calorimetry,2011,pp.145-150.

35. Y.Kar, "Co-pyrolysis of walnut shell and tar sand in a fixed-bed reactor", Bioresource Technology, 2011, pp. 9800-9805.

36. H.Yang, R.Yan, Chen, "Characteristics of hemicellulose, cellulose and lignin pyrolysis", vol,86, Fuel,2011, pp. 1781-1788.
Published By: Blue Eyes Intelligence Engineering

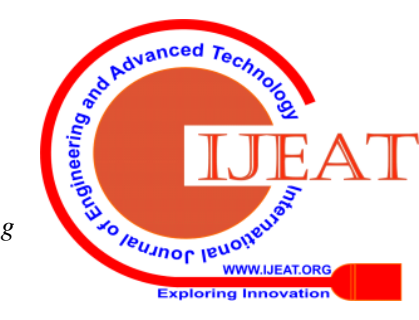

